\title{
Criterios de selección y secuenciación textual en la Enseñanza de Inglés con Fines Específicos: Aspectos teóricos y principios metodológicos
}

\author{
Criteria for the selection and sequencing of texts in English for Specific
}

Purposes classes: Theoretical aspects and methodological principles

Daniela Silvia Moyetta ${ }^{1}$ (1) https://orcid.org/0000-0002-2973-8338

Universidad Nacional de Córdoba, Argentina

Ciudad Universitaria $\cdot$ Córdoba (Argentina)

RESUMEN

Este trabajo tiene como objetivo principal proponer criterios para la selección y la secuenciación de textos especializados en un corpus con fines pedagógicos. Para ello, se construyó un corpus ejemplar de textos especializados de la disciplina Diseño Industrial, que se analizó sobre la base de un enfoque multinivel. Este análisis buscó determinar el grado de especialización de los textos para, luego, establecer criterios de selección y de secuenciación textual de aplicación general, que proporcionen una base fundada para los materiales didácticos usados en las clases de comprensión lectora en el ámbito del Inglés con Fines Específicos.

Palabras clave: Inglés con Fines Específicos, géneros, textos especializados, selección textual, secuenciación textual

ABSTRACT

The main objective of this paper is to propose criteria for the selection and sequencing of specialised texts for teaching purposes. To achieve this aim, a corpus of specialised texts in the field of Industrial Design was constructed and analysed on the basis of a multilevel approach. The goal of the analysis was to determine the degree of specialisation of the texts in order to establish sound criteria for text selection and sequencing, which can help provide the basis for creating the teaching materials used in reading comprehension classes in the field of English for Specific Purposes.

Keywords: English for Specific Purposes, genre, specialised texts, text selection, text sequencing

\section{Introducción}

Comprender el discurso especializado y negociar el acceso y la permanencia en una comunidad disciplinar exige que los interlocutores compartan un conjunto de significados lingüísticos, pragmáticos, retóricos, contextuales y sociocognitivos (Johns, 1997; Cassany y Morales, 2008). De este modo, las comunidades disciplinares se conforman a partir de prácticas de lectura y escritura particulares, con géneros discursivos propios, reconocidos por sus miembros y que condicionan las convenciones para construir conocimientos y los usos del lenguaje (Swales, 1990; Bhatia, 1993; Cassany y Morales, 2008).

El ámbito por excelencia donde se enseña a comprender y a producir los géneros propios de cada comunidad disciplinar es la universidad. En este espacio se espera que los estudiantes lectores sean capaces de buscar, 
interpretar, adquirir y reformular información a partir de textos propios de la disciplina, ya que cada campo de estudio esquematiza, de diferentes maneras, sus saberes y conocimientos a través de lo escrito.

En el contexto académico circulan textos científicos, que son elaborados por investigadores y refieren a temáticas propias de un dominio científico y responden a convenciones y tradiciones retóricas específicas de cada área de conocimiento (Adelstein y Kuguel, 2004; Ciapuscio, 2003). De lo dicho anteriormente, se desprende que cada disciplina posee un repertorio propio de géneros y, por lo tanto, conocer la disciplina exige saber leerlos y producirlos (Cassany, 2008; Parkinson, 2013).

En el ámbito académico-científico, en general, y en el contexto de la Universidad Nacional de Córdoba, Argentina, en particular, circulan textos en inglés, entre otras lenguas. En efecto, y como consecuencia de distintos factores históricos, económicos, políticos y sociales (Crystal, 2003; Grabe y Kaplan, 1996; Mugglestone, 2013, entre muchos otros), el inglés se ha establecido como lengua internacional para el intercambio de conocimientos a nivel mundial (Bhatia, 2008; Grabe y Kaplan, 1996; Hyland, 2009; Johns, A. y Dudley-Evans, 1991; Swales, 1985, 1988, 1990, 2004, entre numerosos autores). Por lo tanto, la comprensión de textos en inglés puede representar un problema complejo para los aprendices de expertos porque éstos no son solo receptores semilegos de textos dirigidos a expertos, sino que, además, en el caso de las universidades de habla no inglesa, son lectores alóglotas insertos en un contexto exolingüe.

Como respuesta, han surgido en las últimas décadas en Argentina cursos de Inglés con Fines Específicos con enfoque en la lectocompresión, cuyo objetivo principal es proveer a los aprendices de especialistas de las herramientas lingüísticas y estratégicas necesarias para abordar textos en una lengua que no es la primera. Es evidente que este abordaje pone en primer plano a los textos, que constituyen el material sobre el cual se organizan estos cursos.

Así, la compilación de textos constituye un instrumento fundamental en la enseñanza de los lenguajes con fines específicos, ya que proporcionan la base de los materiales didácticos con los que se organiza la enseñanza (Hurtado Albir, 2011). Los materiales didácticos empleados en los cursos de lectocomprensión en inglés en el nivel superior requieren textos que sean representativos de las comunidades disciplinares; es decir, los textos deben ser representativos de los géneros que los estudiantes leen en su disciplina universitaria (Bhatia, 1993; Hyland, 2002). El diseño de los materiales de lectura comprensiva, por lo tanto, debe destacar las características de los diferentes géneros prototípicos de las diferentes disciplinas.

El trabajo con corpus de textos especializados con fines pedagógicos conlleva, entonces, la adopción de criterios sólidamente fundados que estén relacionados con la selección textual, en primer lugar, y, en segundo lugar, con la secuenciación con la que serán presentados, según su complejidad.

Una condición, por lo tanto, es que la perspectiva adoptada debe tomar en consideración no solo los aspectos inherentes o microestructurales del texto, esto es, su estructuración y formulación, sino que debe dar cuenta también de la relación de mutuo condicionamiento que éstos mantienen con los aspectos superiores o macroestructurales, o sea, con la situación comunicativa, las funciones y el contenido semántico (Kuguel, 2013). En este contexto, surgen los siguientes interrogantes: ¿cómo se seleccionan estos textos? ¿Cómo se organiza una progresión textual?

Al responder estos interrogantes, con este trabajo se espera contribuir al diseño de materiales de lectura para cursos de Inglés con Fines Específicos, basados en géneros de especialidad, para facilitar su abordaje por parte de aprendices de especialistas. En este caso, nos concentramos en el ámbito de Diseño Industrial. 


\section{Antecedentes}

De particular interés para este trabajo son las propuestas que se han presentado desde el Inglés con Fines Específicos y el Inglés con Fines Académicos, en el marco del diseño de materiales y de programas de estudio.

Bhatia (1991) explora el enfoque basado en el género para el diseño de materiales en Inglés con Fines Específicos. El autor propone usar materiales auténticos que sean "sensibles al contexto" y sobre contenidos temáticos específicos de las disciplinas. Se destaca el rol fundamental del experto en la disciplina, ya que no solo puede proveer información útil de la formación sociolingüística y la estructura cognitiva asociados con el género, sino también puede proveer retroalimentación esencial en varios aspectos del análisis. En esta misma línea, Luzón (2005) sugiere identificar y analizar los distintos géneros que los estudiantes necesitan leer y producir.

Dudley-Evans y St. John (1998) conciben al texto como un "vehículo de información", no como un "objeto lingüístico". Como tal, en su selección para conformar un corpus con fines pedagógicos, se debe tener en cuenta la relación de los textos elegidos con las necesidades de los estudiantes, su autenticidad, la secuencia de actividades/tareas en los textos, y la incorporación gradual de textos cada vez más extensos. Los autores resaltan, además, que se debe tomar en cuenta a los estudiantes y, al igual que Bhatia (1991), a informantes especialistas en las disciplinas al momento de la selección textual. En el mismo sentido, Spector-Cohen, Kirschner y Wexler (2001) proponen el uso de textos auténticos representativos y de temáticas relacionadas con las disciplinas. Sugieren, además, familiarizar a los estudiantes con el léxico y los géneros prototípicos de sus áreas.

Por su parte, Kuzborska (2011) coincide con los autores anteriores en hacer uso de los hallazgos de investigaciones sobre las necesidades de los estudiantes para desarrollar un conjunto de criterios que sean la base del diseño de materiales y también resalta la ayuda de profesores de las materias específicas es imprescindible.

En suma, las intervenciones didácticas propuestas en el marco del Inglés con Fines Específicos e Inglés con Fines Académicos coinciden en un requisito fundamental: contar con una descripción de los géneros que circulan en los ámbitos de especialidad.

En español, y con el objetivo de presentar fundamentos teóricos y describir procedimientos metodológicos que permitan identificar géneros académicos y profesionales, Parodi, Ibáñez, Venegas y González (2010: 256) proponen distintas etapas para recolectar un corpus con el fin de construir una base de datos que sirva como fuente de información para el diseño de materiales.

De particular interés para este trabajo resulta la propuesta presentada por Lapegna (2020) quien, con el objetivo de clasificar y caracterizar textos especializados para seleccionar y secuenciar el material didáctico de cursos de Inglés con Propósitos Específicos, sugiere que los dos ejes determinantes son la relación entre los participantes de la comunicación y el propósito comunicativo de los textos.

Sin embargo, a pesar de la variedad de trabajos en el área, no existen, hasta lo que conocemos, estudios descriptivos publicados que presenten una propuesta que dé cuenta de los pasos a seguir para seleccionar textos representativos de las comunidades disciplinares. 


\section{Algunas precisiones teóricas}

Antes de presentar la propuesta de selección y progresión de textos para la enseñanza del Inglés con Fines Específicos en el contexto universitario, es preciso introducir algunas definiciones de los conceptos centrales para este trabajo, que se enmarca en el enfoque denominado 'cognitivo-comunicativo' de la lingüística textual germana (Heinemann y Viehweger, 1991; Heinemann y Heinemann, 2002) y en la Escuela de Inglés con Fines Específicos. Sobre esta base, explicitaremos las definiciones de esos conceptos.

\subsection{Textos de especialidad}

En el marco de la Lingüística Textual ha existido desde siempre un punto de discordancia entre los especialistas: cómo abordar la problemática de los límites entre los LSP (Languages for Specific Purposes) y la lengua general. Por un lado, se encuentran aquellos que favorecen el trazado de un corte de navaja entre lo general y lo especializado y por otro, se ubican los que consideran que existe un contínuum que va de lo altamente especializado a lo general (Ciapuscio, 2003). En la primera posición aparecen los que tienen una "visión idealizada del quehacer científico y modo de comunicar" (Ciapuscio, 2003). En la segunda posición se encuentran los representantes de corrientes funcionalistas, quienes mantienen que no es posible hacer un corte nítido entre lo general y lo especializado dado lo difuso de sus límites.

Existen nutridas discusiones en la literatura acerca de la posibilidad de separar lo general de lo especializado que han posicionado a los especialistas en dos corrientes principales: los que establecen cortes nítidos y los que postulan la idea del contínuum. Estas discusiones han producido variados intentos de definición de texto especializado.

Cabré (2007: 90-91) sostiene que los textos especializados son:

las producciones lingüísticas, orales o escritas, que se producen en escenarios de comunicación profesional y sirven exclusivamente a una finalidad profesional. Se reconocen los escenarios profesionales por los interlocutores que actúan en la situación, por el tratamiento de una temática relativa al dominio o dominios concernidos por la profesión y por la finalidad de buscar la información del receptor.

Así, las condiciones discursivas de los textos especializados son: el productor o emisor, el o los destinatarios, la organización general de sus estructuras y la selección de unidades de nivel léxico (Cabré 2002: 26).

Ciapuscio y Kuguel (2002: 43) y Ciapuscio (2003: 30) conciben a los textos especializados como:

\footnotetext{
Productos predominantemente verbales de registros específicos, registros que son definidos por los usuarios de los textos, las finalidades y las temáticas. Los textos especiales se refieren a temáticas propias de un dominio de especialidad y responden a convenciones y tradiciones retóricas específicas. Los factores funcionales, situacionales y temáticos tienen su correlato en el nivel de la forma lingüística, tanto en la sintaxis como en el léxico. En el caso de la sintaxis, las unidades y las combinaciones son evidentemente las de la lengua general, pero existe una selección periódica y regular de posibilidades, determinada por el registro y la clase de texto. En cuanto al léxico, la presencia relativa de terminologías es una señal propia y evidente del registro especial, sin embargo, su ocurrencia, densidad y tratamiento por parte de los hablantes es extremadamente variable.
}

La posición de Cabré (2002, 2007), aunque similar en considerar que se debe hablar de "usos especializados" de la lengua y no de "lenguajes especializados", se contrapone con la posición de Ciapuscio y Kuguel, 2002 y Ciapuscio, 2003 en un punto principal: no plantea la distinción "especializado/general", sino que contrapone 
"especializado" a "no especializado", si se toma como punto de análisis los aspectos relativos al mantenimiento del control conceptual.

Por razones de elección y de formación, en este trabajo se toma la definición de textos especializados de Ciapuscio y Kuguel (2002: 43) y Ciapuscio (2003: 30), y se entiende a los textos de especialidad en una concepción gradual, que admite áreas de transición en la delimitación de los textos especializados.

\subsection{Género}

De especial relevancia para este trabajo es la concepción de "género" del Inglés con Fines Específicos, tradición que pone el énfasis en las convenciones lingüísticas y en la estructura retórica. Los estudios de género dentro de esta tradición han estado motivados principalmente por razones pedagógicas, ya que se sostiene que la enseñanza explícita de los géneros relevantes para las diferentes disciplinas puede ayudar a los estudiantes en el ingreso y permanencia en las comunidades discursivas (Bawarshi y Reiff, 2010). Es por este motivo que este trabajo toma la noción de género de Swales (1990: 58):

(...) a class of communicative events, the members of which share some communicative purposes. These purposes are recognised by the expert members of the parent discourse community and thereby constitute a rationale for the genre. This rationale shapes the schematic structure of the discourse and influences and constrains choice of content and style. Communicative purpose is both a privileged criterion and one that operates to keep the scope of a genre as here conceived narrowly focused on comparable rhetorical action. In addition to purpose, exemplars of a genre exhibit various patterns of similarity in terms of structure, style, content and intended audience. If all high probability expectations are realised, the exemplar will be viewed as prototypical by the parent discourse community.

En términos generales, esta definición comprende los aspectos centrales del género: evento comunicativo, propósito comunicativo, convenciones discursivas y nomenclatura de la comunidad de discurso.

\subsection{Tipo textual}

Cuando la clasificación de los enunciados no es parte natural del aprendizaje del hablante, sino que se realiza a partir de herramientas teórico-metodológicas de la lingüística, lo que se proponen son tipos textuales. Los tipos textuales surgen de clasificaciones realizadas sistemáticamente.

Entre las clasificaciones surgidas de los distintos enfoques teóricos, la de Werlich $(1975,1982)$ ha sido considerada como una de las mejores y más fundamentadas (Isenberg 1987, en Ciapuscio, 1994). Werlich distingue cinco prototipos ideales, que están estrechamente relacionados con las actividades cognitivas llevadas a cabo por los seres humanos. Así, el autor sostiene que: (a) la percepción del espacio, sus ocurrencias y cambios dan lugar a la base textual descriptiva; (b) la percepción del tiempo, sus ocurrencias y cambios constituyen la base narrativa; (c) la comprensión de conceptos generales y de conceptos particulares a través del análisis o síntesis da forma a la base expositiva; (d) el razonamiento, entendido como creación de relaciones de similitud, contraste o transformación entre conceptos, forma la base argumentativa; (e) por último, la planificación de acciones futuras compone la base instructiva o directiva (Ciapuscio, 1994; Hurtado Albir, 2011). Esta clasificación de los textos, según sean predominantemente descriptivos, narrativos, expositivos, o 
argumentativos, constituye uno de los modos básicos de organizar el repertorio verbal que un hablante (adulto) posee.

\subsection{El modelo textual de múltiples niveles}

En lo que respecta al contexto discursivo, se adopta un modelo de la linguiística textual que propone una organización modular de los diversos aspectos cognitivos involucrados tanto en la producción como en la comprensión. Esta tipología de múltiples niveles presentada por Ciapuscio y Kuguel (2002) permite tener en cuenta tanto aspectos microestructurales, que comprenden las partes "menudas" del texto, es decir, aspectos léxicogramaticales, como características macroestructurales: propósito comunicativo, distribución de la información en el texto, principales secuencias textuales, que surgen del contexto comunicativo. La división en niveles le imprime a la tipología una característica fundamental que es la flexibilidad. Así es que la descripción del texto a partir de múltiples dimensiones que interactúan entre sí y que, al mismo tiempo, permiten establecer secuencias ordenadas resulta de relevancia para clasificar los textos en función de su grado de especialización. Las autoras proponen la siguiente tipología:

a) Nivel funcional

\section{a.1. Funciones (expresar / contactar / informar / dirigir)}

a.2. Jerarquía funcional (funciones dominantes / subsidiarias / complementarias)

b) Nivel situacional

b.1. Contexto social, tipo de comunicación y situación ambiental

b.2. Participantes de la comunicación: número, rol social y relación entre ellos

c) Nivel del contenido semántico

c.1. Dominio, subdominio y área temática

c.2. Tema del texto y despliegue temático

c.3. Formas primarias o derivadas

c.4. Perspectiva sobre el tema: teórica / didáctica / aplicada / divulgativa

d) Nivel formal

d.1. Máximas de formulación de la clase textual

d.2. Formas lingüísticas / no lingüísticas

d.3. Recursos sintácticos

d.4. Recursos léxicos

El nivel situacional es el que corresponde a los saberes que un hablante tiene sobre diferentes modelos de situaciones y que se activa para proporcionar una solución a una tarea comunicativa específica. Este nivel está 
conformado por cuatro parámetros específicos: el contexto social, el tipo de comunicación, la situación ambiental y los participantes de la comunicación. En lo que respecta al papel social que pueden cumplir los interlocutores, en este modelo se distinguen distintas categorías, definidas a partir del grado de competencia sobre una determinada área del conocimiento: especialista, semiespecialista, lego. Son las diferencias de competencia en el tema específico las que determinan la simetría o asimetría de relación entre los participantes.

El nivel funcional corresponde al conjunto de conocimientos que los hablantes tienen acerca del rol de los textos en la interacción, su contribución a que las metas comunicativas sociales e individuales se realicen y su aporte a la construcción de relaciones sociales. Se distinguen cuatro funciones básicas -expresarse, contactar, informar y dirigir- concebidas en una relación de inclusión y con zonas de transición y de solapamiento. Los textos son, por lo general, plurifuncionales, pero no todas las funciones tienen la misma jerarquía. Las ilocuciones dominantes, que expresan el propósito del emisor, se apoyan en ilocuciones de apoyo o subsidirias, que aseguran, de manera indirecta, el éxito de las dominantes. Así, la estructura pragmático-ilocutiva de un texto se construye jerárquica y secuencialmente (Gallardo, 2009).

El nivel del contenido semántico se relaciona, principalmente, con el qué incluye el texto y cómo lo incluye; es decir, con la selección de la información y su disposición y organización. En este nivel se contemplan, además, los parámetros de dominio, subdominio, área temática, originalidad y perspectiva temática. La originalidad del contenido permite distinguir entre formas primarias (textos fuente) y formas derivadas. La perspectiva temática hace referencia al punto de vista a partir del cual se trata el tema. En el caso de los textos de especialidad, son relevantes la perspectiva teórica o básica, la perspectiva aplicada, la perspectiva didáctica y la perspectiva divulgativa.

En lo que respecta a cómo se organiza y se dispone la información textual, de particular relevancia para este trabajo resultan los enfoques top-down (o descendentes) de análisis. Más específicamente, el análisis de géneros a través de "movimientos retóricos" (o segmentos textuales) desarrollado originalmente por Swales (1981) para describir la sección introducción de los artículos de investigación científica. El objetivo de este tipo de análisis es describir propósitos comunicativos de un texto a partir de la categorización de las unidades que lo conforman. De gran utilidad resulta, además, la distinción entre secuencias descriptivas, narrativas, expositivas o explicativas y argumentativas (Werlich, 1975, 1982; Adam, 1992) para el estudio del despliegue temático.

Finalmente, el nivel formal hace referencia a la superficie del texto; es decir, a la selección y combinación de recursos verbales y no verbales o lingüísticos y no lingüísticos a los que se recurre para formular al texto. Este nivel comprende las máximas retórico-estilísticas del género discursivo, que orientan la producción y la comprensión textual. Estas normas condicionan, al mismo tiempo, aspectos sintácticos, léxicos y del paratexto icónico.

Cabe aquí resaltar que estos módulos no están desvinculados entre sí;

hay un condicionamiento estrecho y recíproco: los niveles funcional, situacional y semántico del texto determinan los aspectos microestructurales (la distribución informativa, las conexiones sintáctico-semánticas entre las oraciones, la sintaxis y el léxico) y viceversa, los rasgos microestructurales son elementos ineludibles a la hora de describir y explicar el objeto texto en sus niveles más globales (Ciapuscio, 2003: 24).

Por último, la atención a los aspectos microdiscursivos a partir de las secuencias textuales predominantes puede sentar las bases para que su presentación obedezca a criterios lingüísticos sólidamente fundados. 


\section{Propuesta metodológica}

Dado que la preocupación del presente trabajo es dar fundamento teórico a la selección y secuenciación de un corpus $^{2}$ textual con fines pedagógicos, y a partir de las revisiones de estudios previos, se presenta la siguiente propuesta, inspirada en los autores mencionados en las secciones anteriores. En este punto, insistimos en que el objetivo de este corpus no es analizar datos lingüísticos a fin de establecer generalizaciones, sino proveer un análisis ejemplar.

(1) Construir una base de datos con todas las referencias bibliográficas obligatorias y de consulta incluidas en los programas de estudio de la carrera objeto de estudio;

(2) Seleccionar y recolectar los textos, según criterios de frecuencia, relevancia y accesibilidad (Reppen, 2010). Con respecto al criterio de frecuencia, se sugiere elegir títulos que, al menos, aparezcan mencionadas dos veces en el listado de bibliografía. Para establecer la relevancia de los textos en inglés, se recomienda apelar a la información provista por un informante especialista en el contexto de una entrevista no estructurada. En cuanto al parámetro de accesibilidad, se recomienda trabajar con textos que se encuentren disponibles en formato digital.

(3) Analizar los textos, según el modelo de múltiples niveles, para obtener resultados que permitan clasificar los textos en términos de un contínuum cuyos polos varíen entre los textos menos especializados y los textos altamente especializados;

(4) Corroborar los resultados del análisis con expertos del área disciplinar;

(5) Jerarquizar los géneros a partir del orden propuesto por Werlich $(1975,1982)$ : textos de base descriptiva, textos de base narrativa, textos de base expositiva y textos de base argumentativa. En otras palabras, establecer un ordenamiento del material de lectura en términos de secuencias textuales predominantes, en una sucesión de la más simple a la más compleja.

\section{Análisis ejemplar}

En este trabajo, nos concentraremos en el ámbito de Diseño Industrial, disciplina en la que la autora se desempeña como docente de Lectocomprensión en Inglés. La selección del área disciplinar obedece a cuatro criterios: la necesidad de explorar una disciplina diferente a las clásicamente indagadas en idioma inglés, tales como Biología y Medicina; la escasez de estudios de tipo empírico que permitan caracterizar géneros discursivos pertenecientes a esta disciplina; la tradición joven de la disciplina en el ámbito de la Universidad Nacional de Córdoba y la necesidad de una descripción exhaustiva del uso que los diseñadores industriales hacen de la lengua en su ámbito de especialidad, para obtener datos empíricos sólidos que guíen el diseño de herramientas didácticas adecuadas que ayuden a mejorar las prácticas lectoras en lengua inglesa de los estudiantes de la antes mencionada disciplina.

Se buscó recolectar información sobre el material escrito en lengua extranjera que aparece como parte de la bibliografía en las propuestas programáticas de las materias que conforman el plan de estudios de la carrera. Para ello, se confeccionó una ficha en la que constan todas las referencias bibliográficas obligatorias y de

Un corpus es un producto artificial que contiene textos auténticos, seleccionados según criterios precisos para ser utilizados como una muestra de la lengua (Pearson 1999: 22). Pearson, J. (1998). Terms in context. Amsterdam / Philadelphia: John Benjamins Publishing Company. 
consulta incluidas en los programas de estudio. A continuación, se muestra un ejemplo, el cual, con las adaptaciones del caso, se presenta como una referencia que se puede aplicar en otras disciplinas:

\begin{tabular}{l|l|l}
\hline Asignaturas por año de cursado & \multicolumn{1}{|l}{ Bibliografía en Inglés (sí / no) } & Títulos \\
\hline Primer año & & \\
\hline (Nombre de la asignatura) & & \\
\hline
\end{tabular}

Figura 1. Ficha bibliográfica

\begin{tabular}{|c|c|c|}
\hline$\overline{\text { Asignaturas }}$ & Bibliografía en Inglés (sí/no) & Títulos \\
\hline \multicolumn{3}{|l|}{ Primer Año } \\
\hline Introducción al diseño industrial & $\mathrm{NO}$ & \\
\hline Morfología I & Sí & $\begin{array}{l}\text { Caivano, José L. (1993). Appearance } \\
\text { (Cesía) en Construction of Scales by Means } \\
\text { of Spinning Desks. New York: Ed. John } \\
\text { Wiley \& } \\
\text { Sons Inc. }\end{array}$ \\
\hline Sistemas de representación I & $\mathrm{NO}$ & \\
\hline Introducción a la tecnología & NO & \\
\hline$\overline{\text { Matemática }}$ & $\mathrm{NO}$ & \\
\hline$\overline{\text { Ciencias humanas }}$ & $\mathrm{NO}$ & \\
\hline Historia I & $\mathrm{NO}$ & \\
\hline
\end{tabular}

Figura 2: Ejemplo de datos recolectados del primer año de la carrera

Una vez confeccionada la ficha bibliográfica, que abarcó cada año de cursado de la carrera, se procedió a la recolección de los textos. Dado que el objetivo principal en esta etapa fue construir un panorama general de los géneros discursivos escritos prototípicos de la disciplina, el foco de atención estuvo puesto en conseguir los títulos mencionados en los programas de las asignaturas que estuvieran disponibles en la biblioteca de la mencionada unidad académica o en formato electrónico. Encontramos que no todos los títulos consignados en las propuestas programáticas analizadas estaban disponibles en la biblioteca de la Facultad de Arquitectura, Urbanismo y Diseño de la Universidad Nacional de Córdoba, facultad en la que se dicta la carrera de Diseño Industrial, y que solo algunos se encontraban en formato electrónico. Debido a esta dificultad, se decidió llevar a cabo entrevistas in situ a las bibliotecarias, con el objetivo de solicitarles información sobre la bibliografía disponible en idioma inglés y los títulos de mayor consulta en esta lengua. A partir de estos datos, se seleccionaron y analizaron textos con una alta frecuencia de consulta.

Luego de haber construido la base de datos con todas las referencias bibliográficas obligatorias y de consulta escritas en inglés e incluidas en los programas de estudio de la carrera y con el asesoramiento de docentesespecialistas en la disciplina, se seleccionaron los textos detallados a continuación. (Cabe aquí aclarar que esta selección corresponde a la segunda etapa de la propuesta metodológica.)

1. Dorst, K. y Cross, N. (2001). Creativity in the design process: co-evolution of problem-solution. Design Studies, 22, 425-437

2. Phaidon Design Classics (1a. ed.). (2006). Londres: Phaidon Press Limited 
3. Shigley, J. y Mischke, C. (Eds.). (1996). Standard Handbook of Machine Design. Estados Unidos: The McGraw-Hill Companies, Inc.

Una vez conformado el corpus, se llevó a cabo un análisis, para obtener una descripción específica de cada texto aplicando la tipología multinivel de Ciapuscio y Kuguel (2002). En la sección que sigue ilustramos la tipología propuesta, sobre la base de un corpus de textos seleccionados.

\section{Texto 1}

El texto 1 es de circulación periódica y se encuentra restringido al ámbito académico-científico. La comunicación establecida es interna a la disciplina, ya que se trata de una comunicación entre expertos en un campo en particular y sobre un tema en particular. El Journal Design Studies está dedicado a la comprensión del proceso de diseño desde una perspectiva interdisciplinaria. La revista se declara como un espacio dedicado a la publicación de investigaciones relacionadas con el proceso de diseño y con principios, técnicas y procedimientos relevantes para la práctica y la pedagogía del diseño.

En cuanto a los participantes de la comunicación, el texto fue producido por especialistas, miembros de la comunidad científica, que desarrollan actividades de investigación en universidades europeas. El destinatario es, también, parte de la comunidad científica: especialistas lectores de la revista en la que aparece el artículo. Si se presta atención a los indicios lingüísticos, referencias a pertenencias institucionales, referencias bibliográficas y referencias a la publicación en la página de inicio de ésta, advertiremos que los roles sociales entre los participantes que se identifican en el artículo son de productores textuales especialistas a lectores especialistas, por lo que la relación que se establece entre los productores y los destinatarios es simétrica.

Este texto tiene como principal propósito comunicativo informar-exponer acerca de los resultados de una investigación. Los pasajes donde predominan las secuencias expositivo-explicativas permiten distinguir este objetivo. Por otro lado, y debido a que uno de los requisitos de publicación es que los resultados sean originales, este texto también busca persuadir a los lectores del interés de su contenido. Las secuencias argumentativas así lo demuestran.

Las secuencias textuales se encuentran relacionadas con la estructura retórica. Es así que en la Introducción tiende a predominar la secuencia expositivo-explicativa, en la Metodología, la secuencia descriptiva, en los Resultados la secuencia expositivo-explicativa, y en la Discusión y Conclusiones, la secuencia argumentativa. En síntesis "la jerarquía funcional, esto es, cuál es la ilocución dominante y cuál la subsidiaria, determina a la vez que es determinada por la clase textual” (Kuguel, 2007: 54).

El texto objeto de análisis fue producido dentro del dominio del Diseño Industrial y del subdominio del proceso del diseño; la temática se aborda desde la creatividad en el proceso de diseño. Este texto es una forma primaria, ya que se trata de un artículo publicado en una revista especializada. Del ámbito de circulación, también se desprende la perspectiva temática adoptada: teórico-aplicada.

La superestructura textual sostiene su "arquitectura" en secciones que distinguen la manera en la que la información se distribuye. En este nivel, si tomamos en cuenta la propuesta IMRD de Swales (1990), que complementa este análisis, podemos afirmar que la estructura retórica del texto analizado no observa esta convención en su totalidad. Las posibles causas de variación en la estructura retórica de estos textos podrían hallarse en la naturaleza de la investigación, por un lado, y a los requisitos de publicación, por otro. 
El análisis microtextual revela la presencia de elementos prototípicos de la clase textual artículo de investigación científica: vocablos, expresiones y estructuras que indican los movimientos y los pasos retóricos que estructuran al texto, voz pasiva y desagentivación para mostrar objetividad, modalización para atenuar afirmaciones, presencia del autor a través de palabras autorreferenciales o de expresiones que indican afectividad.

Los elementos paratextuales lingüísticos responden a la disciplina a la que pertenece el texto y a la naturaleza de la investigación: títulos resuntivos, con un alto grado de condensación de la información. Los elementos paratextuales icónicos sirven de soporte al relato del proceso de investigación ya que, en su mayoría, se trata de representaciones gráficas que ilustran los elementos usados en la metodología, como por ejemplo design briefs y bocetos.

El tratamiento terminológico es escaso; el vocabulario disciplinar no se despliega ni se explica. Si bien a simple vista los vocablos y frases utilizados pueden parecer de interpretación sencilla debido a que también se emplean en el discurso cotidiano (por ejemplo, design), éstos adquieren un valor especializado por el uso específico que se hace de ellos dentro en un campo de especialización en particular (Cabré, 2002).

En síntesis, el análisis de la superficie textual demuestra que los factores funcionales, situacionales y temáticos tienen su correlato en el nivel de la forma lingüística, tanto en la sintaxis como en el léxico (Ciapuscio, 2003).

\section{Texto 2}

El contexto de circulación de este texto es el ámbito profesional, es decir, el espacio en el que se ejecuta la actividad profesional de un diseñador industrial. Los indicios más evidentes de esto se encuentran en el paratexto lingüístico, más precisamente en el prefacio y en las biografías de los autores. Este texto también se emplea en el ámbito formal de la enseñanza y del aprendizaje, porque constituye una de las fuentes de consultas frecuentes en la Facultad de Arquitectura, Urbanismo y Diseño Industrial de la Universidad Nacional de Córdoba ${ }^{3}$.

La comunicación que se establece entre los participantes es interna; tiene lugar dentro de la disciplina del diseño entre expertos en el área, de esta manera, el destinador es un grupo de especialistas en diferentes áreas del diseño industrial. Los destinatarios son lectores expertos, que comparten conocimientos con los productores textuales sobre los objetos presentados en el texto. Prueba de esto son los datos que aparecen en la sección biografía de los autores y en el prefacio. De este modo, la relación que se establece entre destinador y destinatario es simétrica.

El tex to consta, básicamente, de descripciones de productos creados por destacados diseñadores. Este libro puede considerarse una colección de "reseñas de productos/objetos". Vale aquí aclarar y resaltar que la "reseña" es un género prototípico del diseño industrial que contiene la descripción de diferentes aspectos de un objeto y la justificación de las soluciones técnicas adoptadas en el proceso de diseño. En las reseñas se destacan, además, las características que le confieren una apariencia propia al objeto/producto. En consecuencia, la función dominante y las subsidiarias de este texto están determinadas por el propósito comunicativo principal que es presentar proyectos que se consideren innovadores y sustentables como modo de responder a los desafíos medioambientales actuales. Por este motivo, los productores textuales se apoyan en secuencias descriptivas,

Según la información provista por las bibliotecarias de la mencionada unidad académica. 
narrativas, expositivas y argumentativas para transmitir un mensaje sobre lo que se considera esperable o deseable en las creaciones que respeten la sustentabilidad.

En este punto se hace necesario detenerse en la función central que las secuencias descriptivas cumplen en las reseñas de productos. Describir supone la puesta en movimiento de procesos perceptivos del sujeto, quien actualiza en el discurso las dimensiones cognoscitiva (experiencia de ver y saber) y afectiva (experiencia sensible) (Filinich, 2003). En las reseñas de productos/objetos, entonces, la operación descriptiva sirve un doble propósito: ordenar ciertos aspectos que se desean destacar y dirigir la percepción del receptor.

Este texto fue producido en el subdominio del diseño industrial de objetos/productos y trata sobre diseños fabricados en forma industrial de gran valor estético y calidad sustentable. Respecto a la originalidad, el texto constituye una forma primaria. Si se tiene en cuenta el carácter primario del escrito, se puede decir que la perspectiva temática es teórica.

Si bien se encuentran partes textuales estandarizadas, como por ejemplo prefacio, índice de autores e índice de objetos, la información, en general, no se presenta organizada, según lo propone Ibáñez (2008) para el género "texto disciplinar". Se puede decir, entonces, que el texto objeto de análisis se organiza en partes libres, cuyos títulos presentan el contenido de cada sección. Más precisamente, los títulos cumplen la función de presentar al objeto que seguidamente se detalla.

Como se mencionó anteriormente, este texto está conformado por un conjunto de "reseñas de productos/objetos". De ahí la necesidad de hacer referencia, también, a la estructura de este género. Para ello, se parte del abordaje propuesto por Caballero Rodríguez (2001) para las "reseñas arquitectónicas". Según esta autora, una reseña arquitectónica es un género que se caracteriza por estar constituido por textos breves cuyos propósitos comunicativos principales son describir y evaluar un producto, visual o verbal, a una audiencia. Además de la evaluación, existen otras características que le dan forma a este género: la perspectiva (subjetividad) del productor textual con respecto a la descripción del objeto, el rango de artefactos que se examinan y sus respectivos consumidores y la promoción del consumo de esos artefactos. En cuanto a su organización textual, una reseña arquitectónica consiste en tres partes: introducción, descripción y evaluación. La introducción, generalmente, funciona como contexto de apoyo para la descripción y la evaluación. En este sentido, la manera en la que el escritor construye la introducción impacta en el resto de la reseña: éste tiende a destacar o a minimizar diferentes aspectos de un edificio/una obra según su punto de vista.

El análisis de la superficie textual permitió comprobar la presencia de elementos paratextuales lingüísticos e icónicos característicos de las reseñas de diseño que forman parte del texto analizado: títulos que capturan las características que se desean resaltar de los objetos/productos presentados y fotografías que contribuyen a ilustrar lo que se pretende transmitir de ese producto. El entramado de secuencias textuales argumentativas, expositivas, explicativas y narrativas, junto con los recursos sintácticos característicos de éstas, contribuyen a la creación de un discurso prototípico de la disciplina en cuestión, cuya comunidad está "entrenada para leer imágenes y palabras" (Caballero Rodríguez, 2006: 11). Es posible afirmar, entonces, que en las reseñas de productos/objetos, el sistema verbal y el sistema visual se combinan para construir significados. Por ende, los elementos icónicos se alejan de la perspectiva tradicional que los concibe como "elementos auxiliares" (Alvarado, 2006) para adquirir un rol más central. Aquí las imágenes visuales no solo permiten sintetizar el contenido del texto, sino que además promocionan el producto/objeto descripto. 
Finalmente, los recursos lingüísticos empleados reflejan la importancia que la información visual tiene en este texto. Se recurre a frases nominales extensas, que contienen vocablos de la especialidad, para identificar y destacar características inherentes a los objetos a los que hacen referencia.

\section{Texto 3}

En lo que respecta al contexto social, la circulación de este texto se encuentra restringida al ámbito académicoprofesional y sirve como texto de consulta para los estudiantes y para profesionales.

El tipo de comunicación que se establece en el texto es interno, ya que se trata de una comunicación entre estudiantes o profesionales y expertos. Así, el objetivo principal es introducir una temática a un público amplio que tiene la necesidad de obtener, mejorar o actualizar su conocimiento sobre este tema en particular.

En cuanto a los participantes de la comunicación, los productores textuales se posicionan como expertos en la temática que se desarrolla. Esta evidencia se encuentra en los datos provistos en el paratexto lingüístico. Por su parte, los destinatarios son semilegos, estudiantes del nivel superior, o profesionales. Prueba de ello son las referencias específicas al público destinatario en los prefacios. La relación que se establece entre productor y destinatarios es, entonces, asimétrica.

Este texto tiene como principal meta comunicativa informar-exponer acerca de un tema en particular. Las secuencias expositivo-explicativas permiten dar cuenta de este objetivo. La función subsidiaria de este texto es comandar-evaluar, porque se presentan conocimientos que se suponen establecidos y está destinado, primordialmente, a la transmisión de éstos. Con respecto a la originalidad, el texto 3 es una forma derivada, cuya perspectiva temática es didáctica, tal como lo manifiestan los indicios lingüísticos.

Este texto presenta un ordenamiento estandarizado de la información, según la propuesta de Parodi (2008) para el género "manual universitario", puesto que en éste se pueden identificar las tres partes retóricas fundamentales de un manual: Preámbulo, Conceptualización y Ejercitación y Corolario. Cada una de estas partes se realiza en diferentes subpartes o segmentos textuales. Cabe aquí resaltar que, si bien los segmentos textuales descriptos por el autor antes mencionado aparecen en el texto analizado, su ocurrencia y disposición varía. Estas subpartes se manifiestan en secuencias predominantemente expositivo-explicativas.

El análisis microtextual (o de la superficie textual) permitió comprobar la presencia de elementos paratextuales lingüísticos e icónicos que contribuyen a configurar la trama expositiva-explicativa que caracteriza a los manuales, con el fin de reformular conocimientos especializados para hacerlos más inteligibles y de esta manera acercarlos al público destinatario. Es así que se recurre a títulos resuntivos que anticipan y condensan el contenido de las diferentes secciones de los capítulos, figuras, gráficos.

Con respecto a los recursos sintácticos, aparecen tiempos verbales propios de la explicación, como el presente para dar carácter atemporal a los enunciados y relaciones lógicas causales, ilativas y finales (Bassols y Torrent, 1997) y así lograr máxima claridad en los enunciados. Al mismo tiempo, el autor pone en marcha estrategias discursivas para alcanzar la comprensión por parte del receptor. En consecuencia, aparecen ejemplos, paráfrasis y definiciones que sirven de apoyo al discurso. 
Finalmente, y con respecto a los recursos léxicos, se recurre a diferentes operaciones de tratamiento terminológico, como definiciones, reformulaciones y ejemplos, con el fin de adecuar su contenido a públicos con distinto nivel de conocimiento.

De este modo, y a partir de los resultados del análisis textual, una posible jerarquización, que vaya de lo más simple a lo más complejo, en un corpus con fines pedagógicos en el área del Diseño Industrial sería la siguiente: texto 3 (Manual Universitario), texto 2 (Libro Disciplinar) y texto 1 (Artículo de Investigación). Coincidimos, así, con Lapegna (2020) que sugiere secuenciar el material pedagógico comenzando con géneros cuyo propósito comunicativo es didáctico.

\section{Conclusiones}

En primer lugar, creemos que iniciativas como ésta ponen de relieve la importancia de contar con herramientas teórico-metodológicas de la lingüística para establecer criterios fundados de selección y secuenciación textual en el diseño de materiales.

En segundo lugar, y desde una perspectiva aplicada, estamos convencidas de que la delimitación de criterios de selección y secuenciación textual en corpus con fines pedagógicos sólidamente fundados favorece la elaboración de actividades de lectura auténticas, con propósitos comunicativos reales.

Finalmente, el establecimiento de criterios fundados de selección textual, basados en el texto como un todo complejo en el que lo formal, esto es, el nivel "micro", se encuentra estrechamente ligado a los aspectos mayores o "macro" puede contribuir a sentar las bases para diseñar materiales destinados a la práctica pedagógica en el ámbito del Inglés con Fines Específicos (Kuguel, 2013).

Aquí hemos presentado una propuesta de selección y de progresión textual en un corpus especializado con fines pedagógicos y la hemos ilustrado con un análisis textual en una disciplina en particular. Dado lo complejo del objeto de estudio texto, esta propuesta no debe entenderse como acabada. Somos conscientes de la necesidad de profundizar el análisis y de ampliar el corpus textual, a los fines de realizar ajustes y generalizar los resultados.

\section{Declaration of conflicting interests}

The author(s) declared no potential conflicts of interest with respect to the research, authorship, and/or publication of this article.

\section{Funding}

The author(s) received no financial support for the research, authorship, and/or publication of this article.

\section{About the authors}

Daniela S. Moyetta, Profesora de Inglés especializada en Inglés con Fines Específicos; Magíster en Inglés con Orientación en Lingüística Aplicada por la Facultad de Lenguas, Universidad Nacional de Córdoba, 
Argentina. Docente de Inglés con Fines Específicos en la Facultad de Ciencias Exactas, Físicas y Naturales y en la Facultad de Arquitectura, Urbanismo y Diseño Industrial de la Universidad Nacional de Córdoba, Argentina. Investigadora Categoría III en el Programa de la Secretaría de Ciencia y Tecnología, SECyT.

\section{Acknowledgements}

Mi agradecimiento póstumo a la Dra. Inés Kuguel, con quien inicié mi trabajo de investigación en criterios de selección y secuenciación textual en corpus especializados con fines pedagógicos.

\section{Referencias}

Adam, J. (1992). Les textes: types et prototypes. En Ciapuscio, G. (1994). Tipos textuales. Buenos Aires: Eudeba.

Adelstein, A. y Kuguel, I. (2004). Los textos académicos en el nivel universitario. Universidad Nacional de General Sarmiento.

Alvarado, M. (2006). Paratexto. Buenos Aires: Eudeba.

Bassols, A. y Torrent, M. (1997). Modelos textuales. Barcelona: Octaedro.

Bawarshi, A. y Reiff, M. J. (2010). Genre: An introduction to history, theory, research and pedagogy. Indiana: Parlor Press y The WAC Clearinghouse.

Bhatia, V. (1991). A genre-based approach to ESP materials. World Englishes, 10, 153-166.

Bhatia, V. (1993). Analysing genre. Language use in professional settings. Londres: Longman.

Bhatia, V. (2008). Genre analysis, ESP and professional practice. English for Specific Purposes, 27(2), 161-174.

Caballero Rodríguez, M. (2001). Evaluation and rhetorical structure: the case of building reviews in the discourse of architects. En J. Palmer; S. Posteguillo y I. Fortanet (Eds.), Discourse analysis and terminology in languages for specific purposes (pp. 445-451). Publicaciones de la Universidad Jaume I.

Caballero Rodríguez, M. (2006). Re-viewing space. Figurative language in architects' assessment of built space. Berlin/New York: Mouton de Gruyter.

Cabré, M. T. (2002). Textos especializados y unidades de conocimiento. En J. García Palacios y M. T. Fuentes Morán (Eds.), Texto, terminología y traducción (pp. 15-36). Salamanca: Ediciones Almar.

Cabré, M. T. (2007). Construir un corpus de textos de especialidad: condiciones y posibilidades. En M. Ballard y C. PineiraTresmontant (Eds.), Les Corpus en Linguistique et traductoligie (pp. 89-106). Francia: Artois Presses Université.

Cassany, D. (2008). Taller de textos. Leer, escribir y comentar en el aula. Buenos Aires: Paidós.

Cassany, D. y Morales, O. (2008). Leer y escribir en la universidad: Hacia la lectura y la escritura crítica de géneros científicos. Revista Memoralia, Universidad Nacional Experimental de los Llanos Ezequiel Zamora (Unellez), Cojedes: Venezuela.

Ciapuscio, G. (1994). Tipos textuales. Buenos Aires: Eudeba.

Ciapuscio, G. (2003). Textos especializados y terminología. Barcelona: Instituto Universitario de Lingüística Aplicada, Universidad Pompeu Fabra.

Ciapuscio, G. y Kuguel, I. (2002). Hacia una tipología del discurso especializado: aspectos teóricos y aplicados. En J. García Palacios y M. T. Fuentes Morán (Eds.), Texto, terminología y traducción (pp. 37-73). Salamanca: Ediciones Almar.

Crystal, D. (2003). English as a global language (2da ed.). Cambridge: Cambridge University Press. 
Dudley-Evans, T. \& St John, M. (1998). Developments in English for specific purposes. A multi-disciplinary approach. Cambridge: Cambridge University Press.

Filinich, I. (2003). Descripción. Buenos Aires: Eudeba.

Gallardo, S. (2009). La estructura ilocutiva y la distinción entre géneros discursivos. En Ciapuscio, G. (Ed.), De la palabra al texto. Estudios lingüísticos del español (pp.129-162). Buenos Aires: Eudeba.

Grabe, W. \& Kaplan, R. (1996). Theory and practice of writing. London: Addison Wesley Longman.

Heinemann, W. \& Viehweger, D. (1991). Textlinguistik. Eine Einführung. Tübingen: Niemeyer.

Heinemann, M. \& Heinemann, W. (2002). Grundlagen der Textlinguistik. Tübingen: Max Niemeyer Verlag.

Hurtado Albir, A. (2011). Traducción y traductología (5ta Ed.). Madrid: Ediciones Cátedra.

Hyland, K. (2002). Genre: language, context, and literacy. Annual Review of Applied Linguistics, 22, 113-135.

Hyland, K. (2009). Academic discourse. English in a global context. Londres: Continuum.

Ibañez, R. (2008). El Texto Disciplinar y el acceso al conocimiento desde el análisis del género: ¿regulación del conocimiento o persuasión? En G. Parodi, (Ed.), Géneros académicos y géneros profesionales: Accesos discursivos para saber y hacer (pp. 219246). Valparaíso: Ediciones Universitarias de Valparaíso. Pontificia Universidad Católica de Valparaíso.

Isenberg, H. (1987). Cuestiones fundamentales de tipología textual en Ciapuscio, G. (1994). Tipos textuales. Buenos Aires: Oficina de Publicaciones UBA.

Johns, A. (1997). Text, role and context: Developing academic literacies. Nueva York: Cambridge University Press.

Johns, A. y Dudley-Evans, T. (1991). English for Specific Purposes: International in Scope, Specific in Purpose. TESOL Quarterly, 25(2), 297-314.

Kuguel, I. (Noviembre de 2013). Los géneros, los tipos y las secuencias textuales como instrumentos para la enseñanza de ILE. VII Congreso Internacional de la Cátedra UNESCO. Córdoba, Argentina.

Kuguel, I. (2007). La semántica del léxico especializado: los términos en textos de ecología. (Tesis Doctoral no publicada). Universidad de Buenos Aires, Buenos Aires.

Kuzborska, I. (2011). Teachers' decision-making processes when designing EAP reading materials in a Lithuanian university setting. Journal of English for Academic Purposes, 10, 223-237.

Lapegna, M. (2020). Discurso académico escrito en el nivel universitario: clasificación y caracterización de los textos especializados en cursos de Inglés con Fines Específicos. RASAL, 79-109.

Luzón, M. (2005). Aplicación del concepto de "colonia de géneros" a la enseñanza de Inglés para Fines Específicos. Ibérica. 10, 133144.

Mugglestone, L. (2013). The Oxford history of English. Oxford: Oxford University Press.

Parkinson, J. (2013). English for science and technology. En B. Paltridge \& S. Starfield (Eds.), The handbook of English for Specific Purposes (pp. 154-173). U.K.: John Wiley \& Sons, Inc.

Parodi, G. (2008). Géneros académicos y géneros profesionales: accesos discursivos para saber y hacer. Valparaíso: Ediciones Universitarias de Valparaíso. Pontificia Universidad Católica de Valparaíso.

Parodi, G.; Ibánez, R.; Venegas, R. y González, C. (2010). Identificación de géneros académicos y géneros profesionales: Principios teóricos y propuesta metodológica. En Parodi, G. (Ed.), Alfabetización académica y profesional en el siglo XXI: Leer y escribir desde las disciplinas. Chile: Editorial Planeta Chilena.

Reppen, R. (2010). Building a corpus: What are the basics? En A. O'Keefe y M. McCarthy Eds.), The Routledge handbook of corpus linguistics (pp. 31-38). Londres: Routledge.

Spector-Cohen, E.; Kirschner, M. y Wexler, C. (2001). Designing EAP reading courses at the university level. English for Specific Purposes, 20, 367-386.

Swales, J. (1981). Aspects of article introductions. Aston ESP Reports No. 1. The University of Aston in Birmingham.

Swales, J. (1985). English as the international language of research. RELC Journal, 16(1), 1-7.

Swales, J. (1988). Discourse communities, genres and English as an international language. World Englishes, 7(2), 211-220. 
Swales, J. (1990). Genre analysis. English in academic and research settings. Cambridge: Cambridge University Press.

Swales, J. (2004). Research genres. Explorations and applications. Cambridge: Cambridge University Press.

Werlich, E. (1975). Typologie der texte. Munich: Fink.

Werlich, E. (1982). A text grammar of English. Heidelberg: Quelle \& Meyer. 